\title{
The Principle of Brevity in Simultaneous Interpreting
}

\author{
Wang Zheng \\ Tan kah Kee College, Xiamen University, Fujian Province, 361005, China \\ 489406596@qq.com
}

\begin{abstract}
Simultaneous interpreting is a complex cognitive activity that requires the interpreter to listen to what the speaker says and render it immediately into another language, listen to the speaker's next message, store the message in memory before retrieving it again for translation, and monitor his or her own output, all at the same time. To overcome the cognitive overloads that are often inevitable during this process, interpreters employ a variety of strategies. This paper introduces the principle of brevity as an effective strategy to overcome the cognitive overloads and the role it plays in Simultaneous interpreting.
\end{abstract}

Index Terms - Simultaneous interpretation, principle, brevity

\section{Introduction}

Simultaneous interpreting is a complex cognitive activity that requires the interpreter to listen to what the speaker says and render it immediately into another language, listen to the speaker's next message, store the message in memory before retrieving it again for translation, and monitor his or her own output, all at the same time. To overcome the cognitive overloads that are often inevitable during this process, interpreters employ a variety of strategies such as; anticipation, linearity, summarization and omission.[1].

One strategy that has proved effective by many studies is the principle of brevity. In this principle of linguistic communication, language users consciously or unconsciously allow minimal effort in speech comprehension and production. This approach favors the least possible semantic description to convey the message, which reduces the amount of time and effort involved in encoding and decoding.

Generally speaking, brevity-related strategies include abbreviation, code mixing, simplification, omission, generalization and approximation.

These strategies are adopted by interpreters for two purposes: One is to make efficient representation by seeking brevity of expression wherever possible and deliberately deciding not to reformulate some reducible elements that do not carry any communicative information or have been manifested to the audience through other communicative channels; the other is to contain loss of information to the minimum degree by rendering as much essential information and sacrificing the minor or secondary elements when the interpreter is unable to produce complete interpretation stress[2].

\section{Why the principle of brevity is helpful in SI}

\section{1 the effort model}

Simultaneous interpretation (SI) can be modeled as a process consisting of the three efforts, namely the listening and analysis effort $\mathrm{L}$, the short term memory effort $\mathrm{M}$, and the speech production effort $\mathrm{P}$, plus a coordination effort $\mathrm{C}$, which is required to coordinate the three other Efforts:

$$
\text { (1) } S I=L+P+M+C
$$

At any point in time, each effort has specific processing capacity requirements that depend on the tasks it is engaged in. At any time, one, two, three of the efforts are active simultaneously. The total requirements TR are therefore presented as a sum of the individual requirements (though not necessarily an arithmetic sum, as some resources may be shared:

\section{(2) $T R=L R+M R+P R+C R$}

$\mathrm{LR}$ capacity requirement for $\mathrm{L}$

MR capacity requirement for $M$

PR capacity requirement for $\mathrm{P}$

CR capacity requirement for $\mathrm{C}$

One of the most striking and challenging phenomena in interpreting is its fundamental difficulty for the interpreter. The effort models are used to explain the difficulty.

In order for interpretation to proceed smoothly, the following five conditions have to be met:

\section{(3) $T R>T A$}

TA total available processing capacity

(4) $L R>L A$

LA capacity available for $\mathrm{L}$

(5) $M R>M A$

MA capacity available ro $\mathrm{M}$

$$
\text { (6) } P R>P A
$$

PA capacity available for $\mathrm{P}$

$$
\text { (7) } C R>C A
$$

CA capacity available for $\mathrm{C}[3]$.

The condition 3 deals with the total available capacity. When it is not met, or when the total processing capacity requirements exceed available capacity, problems often occurs.

Brevity favors the least possible semantic description to convey the message, which reduces the amount of time and effort involved in interpreting. By using the principle of brevity, PR is lowered, so TR is lowered as a result. Therefore enough processing capacity is left to focus on dealing with the key information so as to keep TR<TA and to avoid the loss of primary information..

\subsection{The tightrope hypothesis}

Research findings show that many errors and omissions in interpreting are not due to the intrinsic difficulty of the corresponding source-speech segments, but to the interpreters working close to processing capacity saturation. Working near 
capacity saturation makes them vulnerable to even small variations in the available processing capacity for each interpreting component. Most of the time, total capacity consumption is close to the interpreter's total available capacity, so that any increase in processing requirements and any instances of mismanagement of cognitive resources by the interpreter can bring about overload or local attention deficit (in one of the efforts) and consequently deterioration of the interpreter's output. This 'tightrope hypothesis' is crucial in explaining the high frequency of errors and omissions that can be observed in interpreting even when no particular technical or other difficulties can be identified in the source speech. Simultaneous interpreters often seem to work near the saturation level of their cognitive resources, as can be seen in the deterioration of a professional interpreter's performance, even when no difficulty can be identified in the source speech. That is to say if interpreters work well below the saturation level, errors and omissions should occur only when significant difficulties come up in the source speech.

Brevity-related strategies such as abbreviation, code mixing, simplification, omission, generalization and approximation reduce the amount of time and effort involved in interpreting, thus bringing down the total processing capacity consumption. By using brevity related strategies, interpreters work well below saturation level. By working below saturation levels, errors and omissions can be reduced to an extent that interpreting can help listeners get main contents and necessary information. The whole interpreting quality can be improved as a result[4].

\subsection{Experiments of simultaneous interpreting}

A study done by Minhua Liu, Diane L. Schallert and Patrick J. Carroll describes an experiment aimed to determine if performance differences exist in simultaneous interpreting by individuals with similar general cognitive abilities, but different skills specific to the task of simultaneous interpreting. The performance of professional interpreters interpreting from English to Mandarin was compared to that of two groups of student interpreters; beginners and advanced. The results showed that the professional interpreters who were not different from students in their general working memory capacity, but their general interpreting performance outperformed student interpreters. This difference was attributed, at least in part, to the development of specific skills in managing competing demands on limited cognitive resources. One important domain-specific skill observed in this study is the ability to select more important ideas from the speech input under conditions where stringent task demands jeopardize completeness and accuracy of the output.

One thing suggested by the study is that expert interpreters, as a way to circumvent limits of working memory resources, are selective in terms of what to interpret and what not to interpret, which is the core value of the principle of brevity[5].

\section{When can we use this principle to a great extent?}

Situation 1: interpret for a wordy speaker

Wordy sentences use too many useless words that clutter thinking. When the speaker is wordy and always repeat what he or she says, it may interrupt the interpreter's logic thinking and make the flow of interpreting unsteady or even broken. Interpreters may use the principle of brevity to a great extent and select the simplest word possible that conveys the same meaning to avoid redundancy and broken interpreting. In this case, the principle of brevity is the best choice.

\section{Let' s see an example of wordy sentence:}

(1)Wordy: For all intents and purposes, the reason $\mathrm{Mr}$. Smith arrived late for work was due to the fact that he stopped at very many traffic lights that were red in color.

(2)Concise: Mr. Smith arrived late for work because he stopped at many red lights.

Sentence (1) is wordy because we can express this thought using fewer words.

We can see sentence (1) and (2) convey the same meaning, but sentence (2) is much easier to understand. The advice is when an interpreter hears a sentence like the sentence (1), he or she may simplify the sentence and make his or her output as concise as sentence (2) in target language.

If an interpreter tries to interpret literally for every word, he or she may slip into a thinking pitfall and make the output unclear and redundant, or in another situation, he may even miss the primary information when sorting out the logic of useless words.

Situation 2: when most people know the two working languages

If the proportion of target-language listeners in the audience is very small, or most people on the spot know the two working languages, the interpreter may suffer from interference. The reason is that source-language listeners often perceive interpretation as a necessary evil; when the delegates who actually need interpretation are few in number and unimportant for the others, interpretation can be perceived as an unnecessary evil that entails loss of time as well as technical constraints (speed of delivery, seating arrangements, the mandatory use of a microphone, etc.). Delegates may therefore put pressure on the interpreter to be very brief or summarize [3]. The interpreter usually can not get the necessary technical help from all parties concerned, for example, the speaker may not try to slow down the speed of delivery or clarify technical terms and concepts; the organizing parties may not pay attention to equip the interpreter with necessary facilities for simultaneous interpreting; the interpreter is forced to make his output as simple as possible to ensure listeners get the primary information and the meeting go on smoothly.

\section{Situation 3: problem triggers}

The principle of brevity can be used to a great extent under situations where problems arise from an increase in processing capacity requirements.

a. A high rate of delivery of the speech. This is often associate with high density of the speech.

A high rate of delivery means more information must be processed per unit of time. When a speaker delivers his or her speech very fast, more processing capacity is required of the interpreters. This applies to both the listening and analysis 
effort and the production effort, as the interpreter's speech is paced by the speakers.

b. High density of the information content of the speech. In particular, enumerations are dense, as they consist of information elements put next to each other without grammatical or other low-density word groups in between. Prepared speeches read by the speaker are generally devoid of the hesitations and are generally more densely formulated than spontaneous speech. Under such conditions, a speech may be fast and dense, therefore requiring processing of more information per unit of time, and raising capacity requirements beyond what is available to the interpreter.

c. External factors such as the deterioration of the quality of the sound coming through the interpreter's earphones, a noisy channel, or other sources of interference also raise the listening and analysis effort's processing capacity requirement Brevity-related strategies such as abbreviation, code mixing, simplification, omission, generalization and approximation may reduce the amount of time and effort involved in interpreting, thus bringing down the total processing capacity consumption to improve quality. For instance, if the interpreter was content with a simpler reformulation, enough capacity would be left over for the primary information [3].

\section{How to put the principle of brevity into practice?}

(1) brevity in note-taking:

Our short-term memory alone can not retain the amount of information the speaker gives in interpreting. Interpreters need to employ the means of note-taking to help with retaining and retrieval of information. Note-taking is necessary to recall the elements of a five-minute speech. Brevity means noting down the key words and main points only or using abbreviation , for example: "prof" for "professor", "GDP" for "gross domestic product", "dept" for “department”, "esp” for "especially”, etc.

\section{(2) Terminologies preparation in advance}

Terminology is where most practical problems lie in technical and scientific translation. Experienced interpreters should prepared the meanings, logic relations of terminologies in advance and use codes, code switching and mixing, or abbreviations to replace the complicated terms to avoid cognitive overload. By working below saturation levels, errors and omissions can be reduced to a largest extent that interpreting can help listeners get main contents

\section{(3) brevity in linguistic output}

Interpreters should be trained to ensure the brief target language output to convey the main structure and logic of a long sentence or speech.

\section{Conclusion}

Simultaneous interpreting is a particularly challenging task because it involves many language processes competing with each other as the individual strives to produce a reasonably high quality output. The principle of brevity is very helpful in terms of overcoming processing overloads to ensure the high quality output. The point is we should learn to select the primary information and know what to omit. As shown in Minhua Liu' paper, professional interpreters were able to interpret more of the source language input accurately than student interpreters. One thing suggested by this study is that expert interpreters, as a way to circumvent limits of working memory resources, are selective in terms of what to interpret and what not to interpret. This is consistent with what every experienced interpreter knows, i.e. that the skill involves constant judgment and selection, not a mere transposition from one language to the other. This is also the main skill needed for the principle of brevity. We could come to a conclusion that training and experience are needed to make good use of this principle to ensure the high quality output.

\section{References}

[1] Setton, R. Simultaneous Interpretation: A Cognitive-pragmatic Analysis Amsterdam/ Philadelphia: John Benjamins Publishing Company. 1997.

[2] Jie Liang, Erchun Chen, Wenyan Xiao. An Initial Discussion on Simultaneous Interpreting: Principles and Requirements [J/OL.http://www.linguist.org.cn/doc/uc200601/uc20060106.pdf, 2007-12-29]

[3] Gile, D. Basic Concepts and Models for Interpreter and Translator Training. Amsterdam/Philadelphia: John Benjamins Publishing Company, 1995

[4] Gile, D. Testing the Effort Models' tightrope hypothesis in simultaneous interpreting - a contribution. Hermes, Journal of Linguistics, 1999, (23): 153

[5] Liu, Minhua, Diane L. Schallert and Patrick J. Carroll 2004. Working memory and expertise in simultaneous interpreting. Interpreting 2004, $6: 1,19-42$. 